\title{
Course of the Diverticular Disease: What is changing?
}

\author{
Tomica Milasavljevic ${ }^{1}$, Giovanni Brandimarte ${ }^{2}$, Neil Stollman $^{3}$, Giovanni Barbara ${ }^{4}$, Adi Lahat $^{5}$, Carmelo Scarpignato $^{6}$, \\ Angel Lanas ${ }^{7}$, Valerio Papa ${ }^{8}$, Antonio Tursi ${ }^{9}$, Gerardo Nardone ${ }^{10}$
}

\footnotetext{
1) Clinical Centre of Serbia,

University of Belgrade, Serbia;

2) Division of Internal Medicine and Gastroenterology, "Cristo Re” Hospital, Rome, Italy; 3) Division of Gastroenterology, Alta Bates Summit Medical Center, East Bay Center for Digestive Health, 300 Frank H Ogawa Plaza, Oakland, CA, USA; 4) Department of Clinical Medicine, Section of Gastroenterology, "S. Orsola” Hospital, University of Bologna, Bologna, Italy; 5) Department of Gastroenterology, Chaim Sheba Medical Center, Tel Hashomer, Ramat Gan 52651, Israel; 6) Faculty of Medical Sciences, United Campus of Malta, San Giljan, Malta; 7) Digestive Diseases Service. University Clinic Hospital. Aragón Health Research Institute (IIS Aragón), University of Zaragoza School of Medicine. CIBERehd. Zaragoza. Spain; 8) Division of General Surgery, "S. Carlo di Nancy” Hospital, Rome, Italy; 9) Territorial Gastroenterology Service, ASL BAT, Andria, Italy; 10) Department of Clinical and Experimental Medicine, University „Federico II”, Naples, Italy
}

Address for correspondence: Gerardo Nardone, MD, PhD Department of Clinical and Experimental Medicine University "Federico II", Naples, Italy nardone@unina.it

\begin{abstract}
In this session several critical issues in diverticular disease were considered, including "It is symptomatic diverticular disease or irritable bowel syndrome?", "What do determine evolution to diverticulitis, bowel habits alteration or inflammation?", and "Prevention of acute diverticulitis: Is it at all possible?".

The first part compared symptoms and laboratory findings between symptomatic uncomplicated diverticular disease (SUDD) and irritable bowel syndrome (IBS). Although both diseases share same symptoms, and although IBS can occur in patients having diverticulosis, SUDD and IBS can be distinguished using a combination of symptoms and laboratory tools.

The second part of this session debated what are the most important risk factors for the evolution towards acute diverticulitis (AD). Current data seem to exclude a significant role of bowel habits alteration, while inflammation seems to have a stronger role, especially in causing acute diverticulitis recurrence.

The third part analyzed the prevention of acute diverticulitis. In primary prevention mesalazine seems to be useful, while no definite conclusion can be drawn about the use of fiber and rifaximin. For secondary prevention, no drugs can be currently advised. At the same time, surgery should be advised on case-by-case basis.
\end{abstract}

Key words: colonoscopy - colorectal cancer - diverticular disease - segmental colitis associated with diverticulosis.

Abbreviations: AD: acute diverticulitis; ASA: amino-salicylic acid; CRP: C-reactive protein; CT: computer tomography; DD: diverticular disease; IBS: irritable bowel syndrome; NNT: number to-need treatment; QOL: quality of life; RCT: randomized controlled study; SUDD: symptomatic uncomplicated diverticular disease; TNF: tumor necrosis factor.

\section{IS IT \\ SYMPTOMATIC DIVERTICULAR DISEASE OR IRRITABLE BOWEL SYNDROME?}

It is symptom at ic diverticular disease!

SUDD is the term used for patients with abdominal symptoms in the setting of diverticulosis, absent overt infection or inflammation. The prevalence of SUDD is unknown, and clinical similarities with IBS impare our ability to epidemiologically distinguish the two entities, which typically run a benign course with a low incidence of complications. The pathophysiology of SUDD is not completely understood, and multiple factors seem to play contributing roles, including visceral hypersensitivity, altered colonic motility, an altered intestinal microbiota and/or lowgrade chronic inflammation [1].

Historically, fiber has been a mainstay of SUDD treatment, although this practice is based on weak evidence and there was no guidance regarding the type and quantity of fiber needed. The possible role of inflammation in SUDD was the basis of investigating mesalamine compounds as therapeutic option. Notably, in a case series of over 900 patients undergoing surgery for SUDD [2] 76\% of cases had evidence of acute or chronic inflammatory changes. The DIVA study [3] compared 12 weeks of mesalamine $2.4 \mathrm{~g} /$ day with placebo after CT-confirmation of acute diverticulitis (AD), and demonstrated a consistent trend towards decreased symptoms, but was underpowered to detect differences in recurrent diverticulitis. A systematic review of 
6 RCTs [4] reported that mesalamine was more effective than placebo in achieving symptom relief in patients with SUDD, although there was significant heterogeneity among the trials, and a much larger trial [5] recently failed to demonstrate any reduction in symptoms or recurrent diverticulitis. While the evidence seems settled that mesalamine is not effective in preventing recurrent diverticulitis, it's role in symptom control in SUDD remains doubtful. On the assumption that the observed disturbances in the intestinal microbiota in patients with SUDD predispose to inflammation and symptoms, rifaximin has also been studied in SUDD with promising results. In a meta-analysis of 4 RCTs in SUDD [6] the combination of rifaximin with fiber was $29 \%$ more effective than placebo in obtaining symptom relief at 1 year, with a NNT of 3. Also based on presumptive dysbiosis, probiotics have been studied, with some benefit shown in trials involving bacterial strains such as Lactobacillus, Bifidobacterium infantis, and combination products such as VSL\#3, but such trials have been small and uncontrolled. While we wait higher-quality evidence to support this approach [7] the microbiome is likely to be an important target for SUDD therapy in the next years.

\section{It is IBS!}

The definition of SUDD: "abdominal symptoms in the setting of diverticulosis, in absence of overt infection or inflammation" is quite confounding from a clinical point of view. This is because the real prevalence of SUDD is unknown, and clinical similarities with IBS (in both there is abdominal pain and change in bowel habits without macroscopic inflammation), as well as the typically benign course with a low incidence of complications, are too similar to allow a clear distinction between the two entities. Specifically, it is still unclear which is the origin of symptoms in patients with SUDD. For example, two large, community-based studies evaluated patients undergoing routine colonoscopy and having chronic abdominal symptoms $[8,9]$ : both found no evidence of mucosal inflammation either based on immune markers, serum CRP or histopathology in patients with diverticulosis regardless of symptoms $[8,9]$. In addition, the good clinical response to rifaximin may also be a confounding factor, since rifaximin was found effective in symptoms' relief in both diseases $[6,10,11]$. Thus, several physicians prefer to define these symptoms as "IBS-like" rather than "SUDD" [12]. Moreover, chronic abdominal pain in patients with diverticulosis may occur from other conditions than SUDD and IBS, it may be secondary to small intestinal bacterial overgrowth, underlying lactose malabsorption, celiac disease, or other functional gastrointestinal disorders [13].

However, some clinical characteristics [14], together with a higher fecal calprotectin [15] and different histo-pathological findings $[16,17]$ can help differentiate SUDD from IBS.

\section{WHAT DETERMINES THE EVOLUTION TO DIVERTICULITIS?}

\section{Bowel habits alteration}

The spectrum of lesions associated with the progression of DD of the colon includes the development of diverticulitis and its complications, although this is very rare compared with the high prevalence of asymptomatic DD in the Western population. In a cohort of veteran patients undergoing colonoscopy in the US, $4 \%$ of patients with known uncomplicated diverticulosis developed $\mathrm{AD}$ after a median-time-to-event of 7.1 years and a rate of 6 cases per 1000 patient-years [18].

The reasons why some patients with asymptomatic DD progress to $\mathrm{AD}$ are unknown, although several potential factors have been suggested, including host genetics factors, diet, medication, lack of exercise, smoking, and bowel habits (constipation) [19]. The type of diet and exercise have been linked to bowel habits, and also to the development of diverticulitis. A recent meta-analysis of nine randomized controlled trials involving 680 participants showed that exercise improved symptoms of constipation, although due to methodological issues, there was a risk of bias and more studies were needed [20]. In the EPIC study [21] a vegetarian diet and patients taking fiber in the highest quartile among the participants were associated with a lower risk of being hospitalized due to diverticulitis. Similarly, patients performing vigorous exercise had a lower risk of diverticulitis compared to those being inactive $[21,22]$. However, there is no direct evidence that bowel habits or constipation are linked to diverticulitis. In a colonoscopy-based cross-sectional study that analyzed 539 patients with diverticulosis and 1569 controls (without diverticulosis), neither constipation, nor a low-fiber diet were associated with an increased risk of diverticulosis [23]. In another study, of 144 patients detected with asymptomatic DD in a screening colonoscopy study and over a mean follow-up of $7.0 \pm 1.7$ years, $4.2 \%$ developed $\mathrm{AD}$ or diverticular bleeding. No variables, including constipation, collected at baseline colonoscopy were associated with these events [24] In a Norwegian population based study conducted to detect risk factors of hospital admission for $\mathrm{AD}$, increased age, obesity, smoking in females and living in rural areas in males, but not constipation were associated with increased risk [25]. In a small study conducted in Asiatic Korean people, constipation was not associated with the development of rightsided diverticulitis [26].

\section{Inflammation}

In recent years, accumulating data suggest that the inflammatory response associated with SUDD may be chronic, with exacerbations and remissions that may lead to the occurrence of AD. Patients were found to have high levels of fecal calprotectin [27], and high levels of TNFa [28], while histopathologic exam showed signs of low-grade inflammation [29]. Those patients seemed to be at higher risk to develop $\mathrm{AD}$, and several clinical studies demonstrated lower risk to develop $\mathrm{AD}$ when using anti-inflammatory treatments such as 5 ASA [30, 31].

Inflammation may also play a role in $\mathrm{AD}$ recurrence. In most patients, the disease is mild, responds well to antibiotic therapy, and does not recur [32]. However, accumulating data suggest more recently that some patients develop the so-called "SUDD post-AD", and that it is not an isolated shortterm event, but rather a chronic inflammatory process with exacerbations and remissions.

Several observations support the idea of chronic mucosal colonic inflammation. Firstly, patients had high levels of 
fecal calprotectin and serum CRP $[27,33]$. Secondly, the level of inflammatory cytokines, TNFa and IL-6, in the affected mucosa was higher compared with nonaffected mucosa [33, 34]. In addition, the histologic evaluation of the affected mucosa showed significantly higher crypt distortion, increased number of lymphoid aggregates, and increased number of lymphocytes in the lamina propria - suggestive of persistent chronic mucosal inflammation [33, 34]. Moreover, several clinical studies demonstrated clinical improvement using anti-inflammatory treatments such as 5-ASA or treatments modulating bacterial-mucosal cross-talk such as rifaximin and probiotics $[35,38]$.

Patients with SUDD, including patients having SUDD post$\mathrm{AD}$, suffer from a prolonged inflammatory process, resulting in recurrent abdominal pain and high levels of inflammatory markers in blood and stool, and structural changes in the affected tissue with high expression of inflammatory cytokines and inflammatory infiltrates.

Further research is warranted for better understanding of the mechanism behind symptomatic DD and for improving patients' treatment.

\section{PREVENTION OF ACUTE DIVERTICULITIS: IS IT AT ALL POSSIBLE?}

\section{Primary prevention of acute diverticulitis}

Progression from SUDD to diverticulitis is uncommon since the disease course is often benign. According to a prospective, long-term study [39], only $2.5 \%$ of patients with SUDD developed AD after a median follow up of 66 months. Prevention (both primary and secondary) of $\mathrm{AD}$ is challenging. Although there are some studies of medical treatments aimed to reduce the occurrence and recurrence of diverticulitis, most of them are of poor quality, and management is often empirical rather than evidence-based.

A vegetarian diet and a high intake of dietary fiber lower the risk of admission to hospital and of death from DD [21]. Rifaximin in combination with fiber is more effective than fiber alone in preventing $\operatorname{AD}[30,40]$, a trend that is also observed in clinical practice [41]. The therapeutic value of rifaximin was however quite low (number needed to treat $(\mathrm{NNT})=59)$ [30]. Finally, a meta-analysis [42] found that mesalazine also prevented diverticulitis occurrence in patients with SUDD.

\section{Secondary prevention of acute diverticulitis}

After an episode of $\mathrm{AD}$, patients might present with recurrent or smoldering diverticulitis, stricture and fistula and often develop chronic GI and non-GI symptoms [43], that may affect their quality of life $[44,45]$. The infection-associated shifts in gut microbial community and the resulting chronic, low-grade mucosal inflammation may well underlie this socalled post-diverticulitis IBS, which has a pathophysiology similar to post-infection IBS [46]. Therefore, several strategies have been undertaken to prevent recurrence of diverticulitis.

\section{Dietary interventions}

Although the American Gastroenterological Association Institute (AGA) guideline on management of $\mathrm{AD}$ [47] recommends a fibre-rich diet or fiber supplementation in patients with a history of $\mathrm{AD}$, a systematic review [48] found that the evidence for this approach was of "very low" quality.

\section{Pharmacological therapies}

Even though a recent updated meta-analysis did not find a significant difference in the rate of recurrent diverticulitis with mesalazine treatment [49], the mesalazine use after an episode of diverticulitis should not be precluded. Mesalazine treatment resulted in fewer and less severe symptoms than placebo $[3,50]$. In addition, mesalazine supplementation led to faster recovery in patients with acute uncomplicated diverticulitis [51].

Albeit preliminary results were promising [52], no conclusive results can be drawn regarding the use of rifaximin for secondary prevention. However, adding mesalazine to rifaximin (both administered 7 days/month for 12 months) seems to be more effective than rifaximin alone for resolution of symptoms and prevention of diverticulitis [53]. Furthermore, the normalization of the inflammatory indexes was faster with the combined treatment [53]. Although rifaximin use can be considered promising, the AGA guideline does not consider the available evidence to be strong enough to recommend the use of rifaximin for the secondary prevention of diverticulitis [47].

A 2012 systematic review concluded that the evidence on medical therapy to prevent recurrent diverticulitis is poor [54]. Therefore, at that time, no medical recommendation for prevention therapy against relapse could have been made.

\section{Surgery}

Surgery is another option in patients with recurrent, uncomplicated diverticulitis, in selected cases. In the past, surgery was recommended after 2 recurrent attacks and potentially sooner in younger (less than 50 years of age) patients [55]. However, accumulating data on the natural history of the disease have led to the abandonment of this recommendation. Indeed, most complications (except fistulas and obstruction) occur during the first or second episode, and emergency surgery is rarely needed in the setting of recurrent disease [56]. Consistent with AGA technical review [57], morbidity is common after elective resection $(10-15 \%)$ and surgery does not eliminate risk of diverticulitis recurrence (5-8\% risk at 8 years). Therefore, elective surgery is recommended for recurrent, uncomplicated diverticulitis on a case-by-case basis, with consideration of factors such as severity and frequency of attacks, effect on QOL, need for immunosuppression, surgical risk profile and patient preference [56].

\section{REFERENCES}

1. Tursi A. Current and Evolving Concepts on the Pathogenesis of Diverticular Disease. J Gastrointestin Liver Dis 2019;28:225-235. doi:10.15403/jgld-184

2. Horgan AF, McConnell EJ, Wolff BG, The S, Paterson C. Atypical diverticular disease: surgical results. Dis Colon Rectum 2001;44:13151318. doi:10.1007/bf02234790

3. Stollman N, Magowan S, Shanahan F, Quigley EM; DIVA Investigator Group. A randomized con-trolled study of mesalamine after acute diverticulitis: results of the DIVA trial. J Clin Gastroenterol 2013;47:621629. doi:10.1097/MCG.0b013e31828003f6 
4. Picchio M, Elisei W, Brandimarte G, et al. Mesalazine for the treatment of symptomatic uncomplicated diverticular disease of the colon and for primary prevention of diverticulitis:a systematic review of randomized clinical trials. J Clin Gastroenterol 2016;50 Suppl 1:S64-S69. doi:10.1097/MCG.0000000000000669

5. Raskin JB, Kamm MA, Jamal MM, et al. Mesalamine did not prevent recurrent diverticulitis in phase 3 controlled trials. Gastroenterology 2014;147:793-802. doi:10.1053/j.gastro.2014.07.004

6. Bianchi M, Festa V, Moretti A, et al. Meta-analysis:long-term therapy with rifaximin in the management of uncom-plicated diverticular disease. Aliment Pharmacol Ther 2011;33:902-910. doi:10.1111/j.13652036.2011.04606.x

7. Scarpignato C, Bertelé A, Tursi A. Probiotics for the treatment of symptomatic uncomplicated diverticular disease:rationale and current evidence. J Clin Gastroenterol 2016;50 Suppl 1:S70-S73. doi:10.1097/ MCG.0000000000000641

8. Peery AF, Keku TO, Addamo C, et al. Colonic Diverticula Are Not Associated With Mucosal Inflammation or Chronic Gastrointestinal Symptoms. Clin Gastroenterol Hepatol 2018;16:884891.e1. doi:10.1016/j.cgh.2017.05.051

9. Jarbrink-Sehgal ME, Rassam L, Jasim A, et al. Diverticulosis, Symptoms and Colonic Inflammation:A Population-Based Colonoscopy Study. Am J Gastroenterol 2019;114:500-510. doi:10.14309/ajg.0000000000000113

10. Li J, Zhu W, Liu W, Wu Y, Wu B. Rifaximin for Irritable Bowel Syndrome:A Meta-Analysis of Randomized Placebo-Controlled Trials. Medicine (Baltimore) 2016;95:e2534. doi:10.1097/ MD.0000000000002534

11 Alamo RZ, Quigley EMM. Irritable bowel syndrome and colonic diverticular disease:overlapping symptoms and overlapping therapeutic approaches. Curr Opin Gastroenterol 2019;35:27-33. doi:10.1097/MOG.0000000000000499

12. Cremon C, Bellacosa L, Barbaro MR, Cogliandro RF, Stanghellini V, Barbara G. Diagnostic challenges of symptomatic uncomplicated diverticular disease. Minerva Gastroenterol Dietol 2017;63:119-129. doi:10.23736/S1121-421X.17.02370-4

13. Bharoucha AE, Chakraborty S, Sletten CD. Common Functional Gastroenterological Disorders Associated With Abdominal Pain. Mayo Clin Proc 2016;91:1118-1132. doi:10.1016/j.mayocp.2016.06.003

14. Cuomo R, Barbara G, Andreozzi P, et al. Symptom patterns can distinguish diverticular disease from irritable bowel syndrome. Eur J Clin Invest 2013;43:1147-1155. doi:10.1111/eci.12152

15. Tursi A, Elisei W, Picchio M, Giorgetti GM, Brandimarte G. Moderate to severe and prolonged left lower-abdominal pain is the best symptom characterizing symptomatic uncomplicated diverticular disease of the colon: a comparison with fecal calprotectin in clinical setting. J Clin Gastroenterol 2015;49:218-221. doi:10.1097/MCG.0000000000000094

16. Walker MM. Inflammation, Genetics, Dysbiosis, and the Environment: New Paradigms for Diagnosis in Complex Chronic Gut Syndromes. J Clin Gastroenterol 2016;50 Suppl 1:S4-S5. doi:10.1097/ MCG.0000000000000613

17. Barbaro MR, Cremon C, Fuschi D, et al. Nerve fiber overgrowth in patients with symptomatic diverticular disease. Neurogastroenterol Motil 2019;31:e13575. doi:10.1111/nmo.13575

18. Shahedi K, Fuller G, Bolus R, et al. Long-term risk of acute diverticulitis among patients with incidental diverticulosis found during colonoscopy. Clin Gastroenterol Hepatol 2013;11:1609-1613. doi:10.1016/j. cgh.2013.06.020

19. Søreide K, Boermeester MA, Humes DJ, Velmahos GC. Acute colonic diverticulitis:modern understanding of pathomechanisms, risk factors, disease burden and severity. Scand J Gastroenterol 2016;51:1416-1422. Doi:10.1080/00365521.2016.1218536

20. Gao R, Tao Y, Zhou C, et al. Exercise therapy in patients with constipation:a systematic review and meta-analysis of randomized controlled trials. Scand J Gastroenterol 2019;54:169-177. doi:10.1080/ 00365521.2019.1568544

21. Crowe FL, Appleby PN, Allen NE, Key TJ. Diet and risk of diverticular disease in Oxford cohort of European Prospective Investigation into Cancer and Nutrition (EPIC): prospective study of British vegetarians and non-vegetarians. BMJ 2011;343:d4131. doi:10.1136/bmj.d4131

22. Strate LL, Liu YL, Aldoori WH, Giovannucci EL. Physical activity decreases diverticular complications. Am J Gastroenterol 2009;104:12211230.

23. Peery AF, Sandler RS, Ahnen DJ, et al. Constipation and a low-fiber diet are not associated with diverticulosis. Clin Gastroenterol Hepatol 2013;11:1622-1627. doi:10.1016/j.cgh.2013.06.033

24. Sharara AI, Ziade N, Shayto RH, et al. The Natural History of Incidental Colonic Diverticulosis on Screening Colonoscopy. Can J Gastroenterol Hepatol 2018;2018:3690202. doi:10.1155/2018/3690202

25. Jamal Talabani A, Lydersen S, Ness-Jensen E, Endreseth BH, Edna TH. Risk factors of admission for acute colonic diverticulitis in a populationbased cohort study: The North Trondelag Health Study, Norway. World J Gastroenterol 2016;22:10663-10672. doi:10.3748/wjg.v22.i48.10663

26. Kim SY, Oh TH, Seo JY, et al. The clinical factors for predicting severe diverticulitis in Korea:a comparison with Western countries. Gut Liver 2012;6:78-85. doi:10.5009/gnl.2012.6.1.78

27. Tursi A, Brandimarte G, Elisei W, Giorgetti GM, Inchingolo CD, Aiello F. Faecal calprotectin in colonic diverticular disease: a case-control study. Int J Colorectal Dis 2009;24:49-55. doi:10.1007/s00384-008-0595-9

28. Tursi A, Elisei W, Brandimarte G, et al. Musosal tumour necrosis factor $\alpha$ in diverticular disease of the colon is overexpressed with disease severity. Colorectal Dis 2012;14:e258-e263. doi:10.1111/j.14631318.2012.02926.x

29. Tursi A, Brandimarte G, Elisei W, et al. Assessment and grading of mucosal inflammation in colonic diverticular disease. J Clin Gastroenterol 2008;42:699-703. doi:10.1097/MCG.0b013e3180653ca2

30. Bianchi M, Festa V, Moretti A, et al. Meta-analysis: long-term therapy with rifaximin in the management of uncomplicated diverticular disease. Aliment Pharmacol Ther 2011;33:902-910. doi:10.1111/j.13652036.2011.04606.x

31. Tursi A, Brandimarte G, Elisei W, et al. Randomised clinical trial: mesalazine and/or probiotics in maintaining remission of symptomatic uncomplicated diverticular disease - a double-blind, randomised, placebo-controlled study. Aliment Pharmacol Ther 2013;38:741-751. doi:10.1111/apt.12463

32. Kaiser AM, Jiang JK, Lake JP, et al. The management of complicated diverticulitis and the role of computed tomography. Am J Gastroenterol 2005;100:910-917.

33. Lahat A, Necula D, Yavzori M, et al. Prolonged recurrent abdominal pain is associated with ongoing underlying mucosal inflammation in patients who had an episode of acute complicated diverticulitis. J Clin Gastroenterol 2019;53:e178-e185. doi:10.1097/MCG.0000000000000980

34. West AB, Losada M. The pathology of diverticulosis coli. J Clin Gastroenterol 2004;38(5 Suppl):S11-S16. Doi:10.1097/01. mcg.0000124005.07433.69

35. Kruis W, Meier E, Schumacher M, et al; German SAG-20 Study Group. Randomised clinical trial: mesalazine (Salofalk granules) for uncomplicated diverticular disease of the colon - a placebo-controlled study. Aliment Pharmacol Ther 2013;37:680-690. doi:10.1111/apt.12248 
36. Lahner E, Esposito G, Zullo A, et al. High-fibre diet and Lactobacillus paracasei B21060 in symptomatic uncomplicated diverticular disease. World J Gastroenterol 2012;18:5918-5924. doi:10.3748/wjg.v18.i41.5918

37. Colecchia A, Vestito A, Pasqui F, et al. Efficacy of long term cyclic administration of the poorly absorbed antibiotic rifaximin in symptomatic, uncomplicated colonic diverticular disease. World J Gastroenterol 2007;13:264-269. Doi:10.3748/wjg.v13.i2.264

38. Campanini A, De Conto U, Cavasin F, et al. A Primary-Care Interventional Model on the Diverticular Disease:Searching for the Optimal Therapeutic Schedule. J Clin Gastroenterol 2016;50 Suppl 1:S93-S96. doi:10.1097/MCG.0000000000000670

39. Salem TA, Molloy RG, O`Dwyer PJ. Prospective, five-year follow-up study of patients with symptomatic uncomplicated diverticular disease. Dis Colon Rectum 2007;50:1460-1464. doi:10.1007/s10350-007-0226-5

40. Kasturi KS, Mummadi RR, Jaganmohan S. M1695 Cyclical Rifaximin for Symptomatic, Uncomplicated Diverticular Disease: A MetaAnalysis. Gastroenterology 2008;134 (Suppl 1):A-399. doi:10.1016/ S0016-5085(08)61865-X

41. Di Mario F, Miraglia C, Cambiè G, et al. Long-term efficacy of rifaximin to manage the symptomatic uncomplicated diverticular disease of the colon. J Investig Med 2019;67:767-770. doi:10.1136/jim-2018-000901

42. Picchio M, Elisei W, Tursi A. Mesalazine to treat symptomatic uncomplicated diverticular disease and to prevent acute diverticulitis occurrence. A systematic review with meta-analysis of randomized, placebo-controlled trials. J Gastrointestin Liver Dis 2018;27:291-297. doi:10.15403/jgld.2014.1121.273.pic

43. Chabok A, Pahlman L, Hjern F, Haapaniemi S, Smedh K; AVOD Study Group. Randomized clinical trial of antibiotics in acute uncomplicated diverticulitis. Br J Surg 2012;99:532-539. doi:10.1002/bjs.8688

44. Spiegel BM, Reid MW, Bolus R, et al. Development and validation of a disease-targeted quality of life instrument for chronic diverticular disease: the DV-QOL. Qual Life Res 2015;24:163-179. doi:10.1007/ s11136-014-0753-1

45. Cohen E, Fuller G, Bolus R, et al. Increased risk for irritable bowel syndrome after acute diverticulitis. Clin Gastroenterol Hepatol 2013;11:1614-1619. doi:10.1016/j.cgh.2013.03.007

46. Barbara G, Grover M, Bercik P, et al. Rome Foundation Working Team Report on Post-Infection Irritable Bowel Syndrome. Gastroenterology 2019;156:46-58.e7. doi:10.1053/j.gastro.2018.07.011

47. Stollman N, Smalley W, Hirano I; AGA Institute Clinical Guidelines Committee. American Gastroenterological Association Institute
Guideline on the Management of Acute Diverticulitis. Gastroenterology 2015;149:1944-1949. doi:10.1053/j.gastro.2015.10.003

48. Dahl C, Crichton M, Jenkins J, et al. Evidence for Dietary Fibre Modification in the Recovery and Prevention of Reoccurrence of Acute, Uncomplicated Diverticulitis: A Systematic Literature Review. Nutrients 2018;10:E137. doi:10.3390/nu10020137

49. Khan RMA, Ali B, Hajibandeh S, Hajibandeh S. Effect of mesalazine on recurrence of diverticulitis in patients with symptomatic uncomplicated diverticular disease:a meta-analysis with trial sequential analysis of randomised controlled trials. Colorectal Dis 2018;20:469-478. doi:10.1111/codi.14064

50. Parente F, Bargiggia S, Prada A, et al; Gismi Study Group. Intermittent treatment with mesalazine in the prevention of diverticulitis recurrence:a randomised multicentre pilot double-blind placebo-controlled study of 24-month duration. Int J Colorectal Dis 2013;28:1423-1431. doi:10.1007/s00384-013-1722-9

51. Nespoli L, Lo Bianco G, Uggeri F, et al. Effect of oral mesalamine on inflammatory response in acute uncomplicated diverticulitis. World J Gastroenterol 2015;21:8366-8372. doi:10.3748/wjg.v21.i27.8366

52. Lanas A, Ponce J, Bignamini A, Mearin F. One year intermittent rifaximin plus fibre supplementation vs. fibre supplementation alone to prevent diverticulitis recurrence: a proofof-concept study. Dig Liver Dis 2013;45:104-109. doi:10.1016/j. dld.2012.09.006

53. Tursi A, Brandimarte G, Daffina R. Long-term treatment with mesalazine and rifaximin versus rifaximin alone for patients with recurrent attacks of acute diverticulitis of colon. Dig Liver Dis 2002;34:510-515. doi:10.1016/s1590-8658(02)80110-4

54. Unlü C, Daniels L, Vrouenraets BC, Boermeester MA. Systematic review of medical therapy to prevent recurrent diverticulitis. Int J Colorectal Dis 2012;27:1131-1136. doi:10.1007/s00384-012-1486-7

55. Aydin HN, Remzi FH. Diverticulitis: when and how to operate? Dig Liver Dis 2004;36:435-445. doi:10.1016/j.dld.2004.03.001

56. Strate LL, Morris AM. Epidemiology, pathophysiology, and treatment of diverticulitis. Gastroenterology 2019;156:1282-1298.e1. doi:10.1053/j. gastro.2018.12.033

57. Strate LL, Peery AF, Neumann I. American Gastroenterological Association Institute Technical Review on the Management of Acute Diverticulitis. Gastroenterology 2015;149:1950-1976.e12. doi:10.1053/j. gastro.2015.10.001 